\title{
Preisach-Mayergoyz approach to fatigue-induced irreversibility
}

\author{
M. Scalerandi,* M. Nobili, M. Griffa, A. S. Gliozzi, and F. Bosia \\ INFM -Dip. Fisica, Politecnico di Torino C.so Duca degli Abruzzi 24, 10129, Torino, Italy
}

(Received 26 January 2006; published 13 March 2006)

\begin{abstract}
The Preisach-Mayergoyz (PM) approach has been widely used to describe hysteresis in different fields. Among these, various types of reversible hysteretic elastic behavior have been succesfully modeled, inclusing quasi-static, fast and slow dynamics, modulation, and so on. Here, we propose an approach to extend the PM formulation to also treat irreversible phenomena, such as fatigue and damage progression. For this purpose, we introduce a multilevel scheme based on nested PM descriptions. The mathematical formulation is outlined and a phenomenological application presented.
\end{abstract}

DOI: 10.1103/PhysRevB.73.092103

PACS number(s): 62.20.Mk, 07.05.Tp, 62.20.Fe, 81.70.Bt

\section{INTRODUCTION}

The internal structure of a medium affects its elastic properties and evidence has been found of the importance of the nonlinear elastic response to external loading in several materials, such as rocks, ${ }^{1}$ concrete, ${ }^{2}$ structural materials, ${ }^{3}$ ceramics, ${ }^{4}$ and composites. ${ }^{5}$ Typical strain ranges are of the order of $10^{-7}$ to $10^{-5}$. In particular, the type of elastic response of a medium is used to assess its mechanical integrity. ${ }^{6,7}$ The opposite is also true: perturbations inducing large amplitude mechanical waves (e.g., during fatigue cycling) modify the medium structure, leading to damage progression. ${ }^{8-10}$ These two phenomena (nonlinear wave propagation and damage progression), which occur at different strain scales $\left(10^{-7}\right.$ to $\left.10^{-2}\right)$, are usually described in the framework of different models and approaches, even though they are linked by a feedback process and share several common features. Indeed, their physical origin can be, at all scale levels, traced back to the inner complexity of the material, which results in an emergent high level of nonlinearity with irreversible processes leading to large energy dissipation.

To describe the observed phenomenology, several models have been proposed,${ }^{11-13}$ mostly starting from ad hoc defined hysteretic functions. Our approach differs in that we exploit the idea that the inner complexity mentioned above conveys the concept that damage, its dynamics and interaction with elastic waves, are emergent phenomena, deriving from interactions between a large number of simple constitutive units on a lower space scale. The Preisach-Mayergoyz (PM) space $^{14,15}$ seems to be an appropriate general framework for this purpose. Indeed, the PM approach is based on the introduction of simple units, ${ }^{16-21}$ characterized by some local (and weakly nonlocal) interactions that satisfy simple equilibrium and constitutive equations. A complex behavior stems from their interactions, rather than from more complicated constitutive equations introduced by hand.

The models presented so far, however, account only for elastically fully reversible constitutive equations. As such, are inadequate to describe plastic strains such as those occurring during damage progression. Furthermore, the "feedback" between modifications in the microstructure and wave propagation has not yet been described in a PM space framework.

As discussed below, the PM approach can also be extended to deal with irreversible processes, while maintaining a mathematical formulation capable of describing the crossinteractions between the wave and the material microstructure at both large and small strains. For this purpose, we introduce here a "multilevel" PM description, i.e., a sort of hierarchy from an upper level to a lower one, with explicit feedback between the various levels. At each intermediate level a statistical ensemble of hysteretic elements (HE) is defined and the behavior of each HE results from the collective behavior of a statistical ensemble defined on the lower hierarchical level.

Here, we limit ourselves to introduce a formalism based on phenomenological considerations. Indeed, in the past years efforts have been made to explain the physical mechanisms at the basis of elastic hysteresis in solids. ${ }^{22-24}$ In our opinion, a clear comprehension of the phenomena involved remains to be achieved and it is difficult to go beyond a phenomenological approach, which, nevertheless, may be both very simple and accurate enough to support the understanding of the observed phenomenology and the mechanisms involved. ${ }^{25,26}$

\section{PM SPACE DESCRIPTION}

Let us assume we are interested in describing the temporal evolution of a quantity $A(t)$ as a function of $B(t)$. The link between $A$ and $B$, called "constitutive law," depends on a material property $C^{1}(t)$, which is also time dependent (the need for the introduction of the index 1 will become evident later). A PM space description consists in extracting $C^{1}(t)$ from the statistical behavior of a large number $\left(N^{1}\right)$ of simple units, e.g., by a simple average:

$$
C^{1}(t)=\left\langle C_{i}^{1}(t)\right\rangle i=1 \cdots N^{1}
$$

Each unit (i) may be in one of two states (denoted as "open" and "closed" in the following), in which $C^{1}$ assumes the value $C_{o}^{1}$ and $C_{c}^{1}$, respectively. The transition between the states is driven by an external variable $D^{1}(t)$, as depicted in Fig. 1. We define for each unit a parameter pair $\left(D_{c i}^{1}, D_{o i}^{1}\right)$, with $D_{o i}^{1}<D_{c i}^{1}$, so that starting at a given $D^{1}<D_{c i}^{1}$, the unit is in the open state up to $D^{1}=D_{c i}^{1}$, at which point it switches to the closed state. Conversely, when $D^{1}$ decreases, the state changes again at $D^{1}=D_{o i}^{1}$. A given distribution of $\left(D_{c}^{1}, D_{o}^{1}\right)$ 


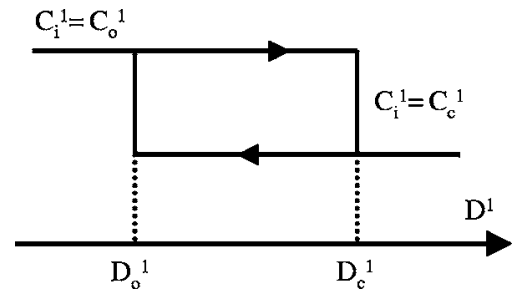

FIG. 1. Bi-state representation of the material property as a function of the driving state variable.

pairs ensures a statistical behavior and accounts for memory effects (for details on this topic, see, e.g., Ref. 25).

Each level of the description through a PM space approach is defined once the driving variable $D^{1}$ has been chosen, and a number of parameters have been defined: The values $C_{o, c}^{1}$, the number of units $N^{1}$ and their distribution $\rho^{1}\left(D_{o}^{1}, D_{c}^{1}\right)$. The parameter $N^{1}$, provided it is large, loses its relevance except in defining the smoothness of the results.

\section{MULTI-LEVEL IMPLEMENTATION}

The multilevel PM description is based on the idea that any of the parameters used in the description at the first level (e.g., $C_{o, c}^{1}$ ) follow a temporal evolution equation driven by a "lower level" material property $C^{2}(t)$. The latter is described in a PM framework identical to the one used for the upper level $\left[C^{2}(t)=\left\langle C_{i}^{2}(t)\right\rangle\right]$, except that the transition between the states $\left(C_{o}^{2}\right.$ and $\left.C_{c}^{2}\right)$ is driven by a variable $D^{2}$. The approach may be repeated on different levels or with different branching. Feedback is then obtained if the variable $D$ at one level corresponds to the output variable $C$ at some other level.

\section{A THREE-LEVEL EXAMPLE}

As an example of implementation, we consider a phenomenological description of damage progression during loading/unloading fatigue cycling. ${ }^{8-10}$ For this purpose, we consider a one-dimensional (1D) material specimen (e.g., a thin bar) subject to a varying external mechanical loading, applied orthogonally to the two surfaces of the bar. The elastic response of the material to the applied load consists of both reversible (e.g., hysteresis in the reversible stress-strain loops $^{9}$ ) and irreversible effects (e.g., plastic softening and residual/irreversible deformations with increasing fatigue $\left.{ }^{8,10}\right)$. To describe the system, we consider a three-level implementation.

Level 1: Following the approach introduced in Ref. 19, we model the specimen as a dynamical system composed of a set of $N$ linear springs, each of which may be in one of two different states, characterized by different rest lengths $l_{r}=l_{0}$ $+\delta_{o, c}$, with $\delta_{o, c}= \pm \delta$ corresponding to $C^{1}$ in the notation adopted above. Each spring switches from one state to the other depending on the stress $\sigma$ it is required to support (positive stress corresponds to compression). Each spring is characterized by a specific $\left(\sigma_{c}, \sigma_{o}\right)$ pair. The first-level state equation, relating stress $\sigma$ and strain $\varepsilon$ ( $A$ and $B$ in the notation used above), is then given by

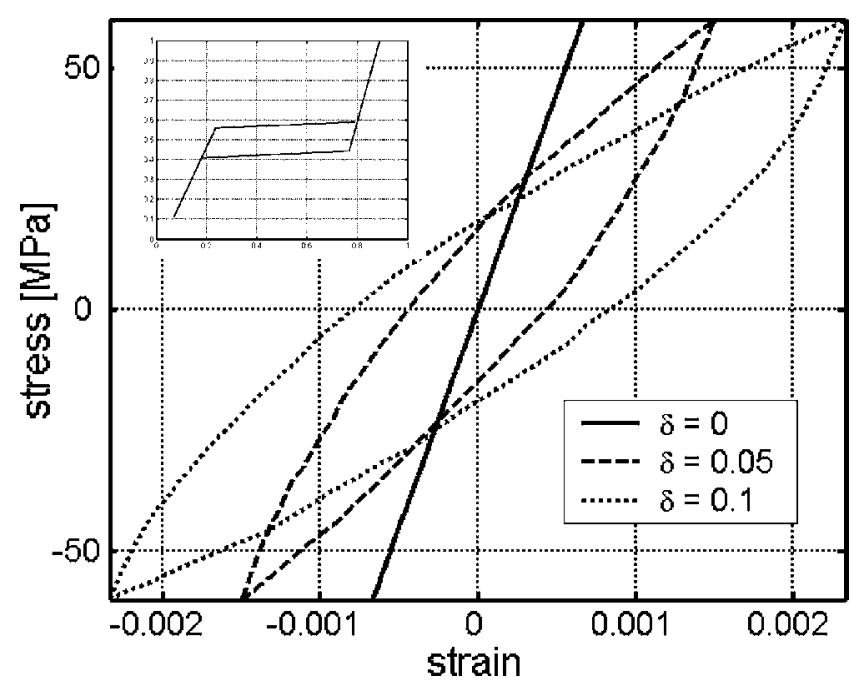

FIG. 2. Stress-strain relation for a chain of 1000 springs with $K=90 \mathrm{GPa}, l_{0}=60 \mu \mathrm{m}$ and different values of $\delta$ (in $\mu \mathrm{m}$ ). The applied stress has the form $\sigma_{\text {ext }}=60 \sin (0.02 \pi t) \mathrm{MPa}$. The behavior of one of the units in the chain is reported in the inset.

$$
\sigma(t)=K \varepsilon(t) \simeq K \frac{\left(l_{r}(t)-l(t)\right)}{l_{r}(t)}
$$

where $l(t)$ and $K$ are the spring length and spring constant. In Fig. 2 we show a stress-strain plot for a chain of 1000 springs and different values of $\delta$. The stress-strain curves for increasing $\delta$ agree well, e.g., with the experimental behavior of alloys and composites with increasing damage (see, e.g., Figs. 9 and 10 in Ref. 10). As in the experiments, the strain is calculated with respect to the average restlength $l_{o}$. The presence of hysteretic loops is recognized to be evidence for various kinds of damage. For instance, the multiplication and propagation of matrix cracks in a composite specimen is known to be reflected in a decrease of the Young's modulus. ${ }^{8}$ At the same time, an increase in the debonding length between matrix and fibers causes an increase in energy dissipation, with consequent increase of the width of the stressstrain loops.

The observed residual strain, i.e., the strain remaining when the applied stress is completely released, is due to the change in rest length with respect to the initial configuration of the $M$ units located in the region $\left(\sigma_{o}<0 ; \sigma_{c}>0\right)$. For an appropriate choice of the initial conditions (for a complete discussion see Ref. 25), these units are in the open state at $t=0$. When compression starts, they switch to the closed state (i.e., more compressed). During unloading, as long as the stress remains positive, they do not switch back to the original state, leading to a positive residual strain (compression), whose value is given by $M \cdot \delta$. As shown in the inset of Fig. 2 , a single spring of the chain follows a different elastic behavior in the two states, while in Ref. 19 only a different restlength was accounted for. The reported data indicate that the parameter $\delta$ is the indicator of both hysteresis and nonlinearity ( $\delta=0$ corresponds to the linear/intact case).

Level 2: To introduce irreversible effects in the model, we assume the parameter $\delta$ to be different for each spring of the 
chain and increasing with time accordingly to the following equation

$$
\dot{\delta}=k \chi
$$

where $k$ is a fixed parameter and $\chi$ is a random number which may assume the value 1 or 0 with probabilities $p_{r}$ and $1-p_{r}$, respectively. The increase of $\delta$ ensures an increase in the nonlinearity/damage. It follows that the probability $p_{r}$ of further damaging should be stress-history dependent. For instance, in composite materials, pullout of the longer fibers during loading promotes debonding of the interfaces, in consequence of the decrease of the interfacial sliding resistance. ${ }^{27}$ Hence, we use a PM space representation for the variable $p_{r}$, assuming it to be given by the average of the values assumed by a large number $N$ of lower level units: $p_{r}=\left\langle p_{r_{i}}\right\rangle$. Each unit at the lower level may be in one of two states: Open state, with $p_{r_{i}}=0$; closed state, with $p_{r_{i}}=p_{1}$. Both tension and compression lead to the same qualitative behavior, particularly in the early stage of damaging. As a consequence, the variable driving the transition between the states is chosen to be the absolute value of the stress supported by the given spring in the chain. Here we neglect that tension is expected to be more effective than compression in inducing damage, leading to asymmetries, which, however, may be easily introduced in our approach. The pair of positive values $\left(\bar{\sigma}_{c}, \bar{\sigma}_{o}\right)$ is specific to each unit. In Fig. 3, we show the values of $p_{r}$ vs stress [Fig. 3(a)] and $\delta$ versus time [Fig. 3(b)] for the central spring in the chain relative to the same case as in Fig. 2 (the applied stress varies sinusoidally with a period of $100 \mathrm{~s}$ ). Note the two-lobe hysteretic loop in the behavior of the damaging probability, with, as expected, the maximum probability of damage occurring in correspondence with the largest applied stress. Also, for a given stress, the probability value is greater in the unloading phase, which reflects the conditioning of the material. A similar behavior is reflected in the temporal evolution of $\delta$, which shows an approximately linear increase of damage with successive plateaux corresponding to the time interval when the applied stress is lowest.

Level 3: A further level of description can be introduced to model the observed feature that damaging is more likely to occur in a previously damaged zone, i.e., where $\delta$ is largest. For instance, it is known that an increase in the debonding length between matrix and fibers in a composite specimen should increase the probability of fiber fracture. ${ }^{28}$ To describe this effect, we assume the lower level parameter $p_{1}$ to be different in each spring of the chain and to be given as the average over $J$ lower scale units $\left(p_{1}=\left\langle p_{1_{i}}\right\rangle\right)$ which may be in one of two states (open state, with $p_{1_{i}}=p_{o}$; closed state, with $p_{1_{i}}=p_{c}, p_{c}>p_{o}$ ). The transition between the two states is driven by the value of the parameter $\delta$ in the corresponding element of the chain. It follows that $p_{1}$ always increases. In Fig. 4 we plot the distribution in the specimen of damage versus time, i.e., the values of $\delta$ along the bar length after successive cycles. The snapshots, in which lighter tones denote larger damage, show that up to about 200 cycles no damage occurs in the specimen. Later, some damaging oc-
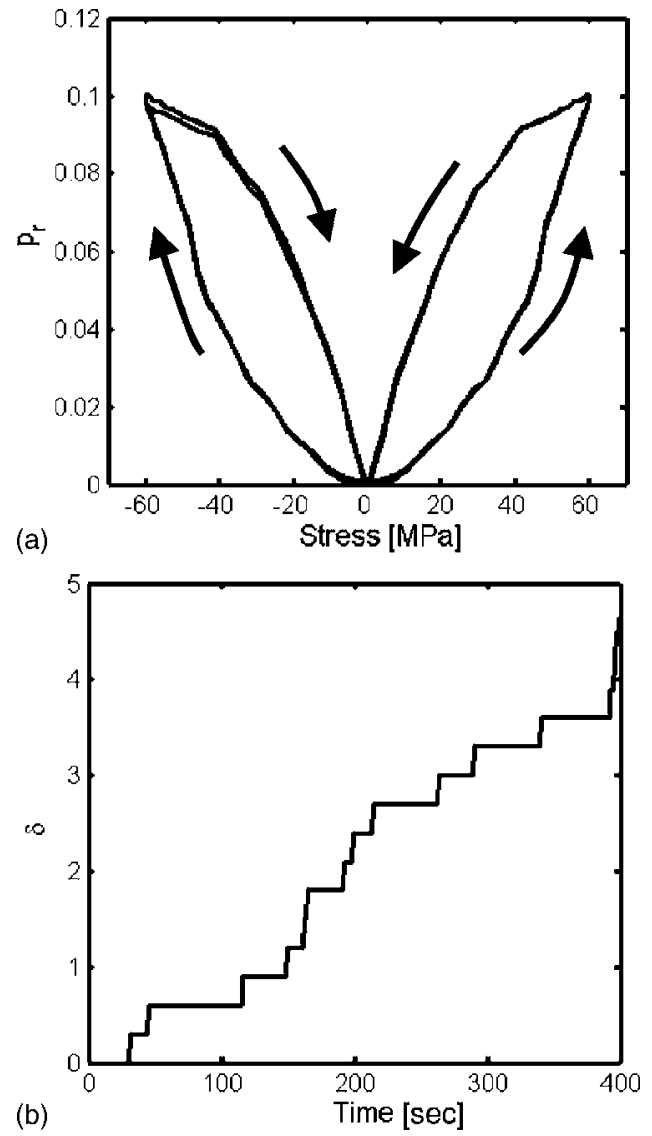

FIG. 3. (a) Damaging probability vs. stress and (b) damage indicator vs time for the central spring of a chain of 1000 springs. $K=90 \mathrm{GPa}, l_{0}=60 \mu \mathrm{m}, M=200, k=0.005 \cdot l_{0}, p_{1}=5 \cdot 10^{-4}$. The applied stress is $\sigma_{\text {ext }}=60 \sin (0.02 \pi t) \mathrm{MPa}$.

curs with a rapid localized increase and noticeable clustering. At later cycles (not reported) damage spreads to the full specimen.

\section{RESULTS}

We finally consider a specific experiment, similar to that reported in Ref. 8 consisting in the measurement of the fatigue properties of an improved $\mathrm{SiC} / \mathrm{SiC}$ composite (HiNicalon ${ }^{\mathrm{TM}} / \mathrm{SiC}$, Young's modulus $=89 \mathrm{GPa}$ ) in air at high temperature. The specimen is subject to successive loading/ unloading cycles with stresses varying between 0 and

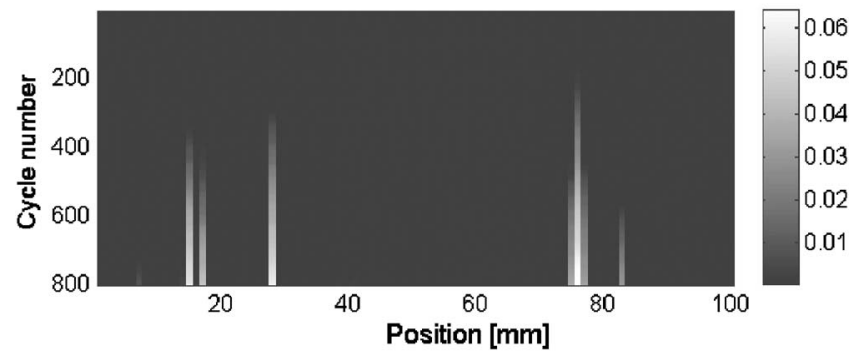

FIG. 4. Distribution of damage during successive loading cycles. Lighter tones denote greater damage. 


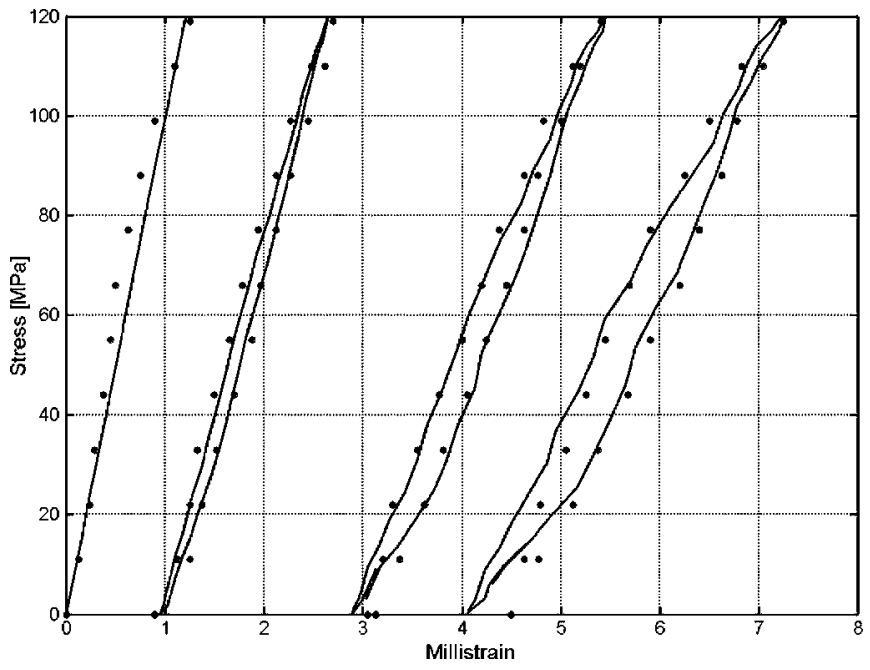

FIG. 5. Comparison of experimental (dots) and theoretical (solid line) stress-strain curves for enhanced $\mathrm{SiC} / \mathrm{SiC}$ composites during fatigue cycling. Experimental data have been extracted from Fig. 5 in Ref. 8.

$120 \mathrm{GPa}$, and the strains are measured during successive cycles. In Fig. 5, the resulting stress-strain curves are shown for a few selected cycles and numerical results (solid lines) are compared with experimental data (dots) taken from Fig. 5(b) in Ref. 8. In the simulations the specimen is assumed to be initially intact, as in experiments. The best choice of parameters to fit the data is the following: first level parameters $K=89 \mathrm{GPa}, l_{0}=60 \mu \mathrm{m}, N=5000$; second level parameters $k$ $=4 \cdot 10^{-4} l_{0}, M=100$; third level parameters $p_{o}=1.5 \cdot 10^{-3}, p_{c}$ $=3.5 \cdot 10^{-3}, J=50 . l_{0}$ may be scaled to adjust the specimen dimension. The agreement between numerical results and experimental data is very good, with the proposed approach capturing the relevant features of the emerging hysteretic loops: the increase in the residual deformation, the fact that the loops are closed and the decrease in slope and increase in width when the number of cycles increases. At the same time, the loops move towards higher strain values (more compressed), in agreement with experimental data. Finally, the fact that each loop is closed is in good agreement with other data (see Fig. 2 in Ref. 9), showing the repeatability of immediately successive loops.

\section{CONCLUSIONS}

We have presented an approach to describe damage progression and other reversible and irreversible effects affecting the elastic properties of nonlinear media. The method proposed consists in an extension of the well known Preisach-Mayergoyz approach, introducing a multilevel description of the medium in which nested PM spaces are used. An application has been presented to clarify the procedure and to show its capability to adapt to real experimental situations. We have given a general formulation of the model, with some qualitative physical justifications for the introduced formalism and the obtained results. The approach seems to us sufficiently general to allow its application to a number of different physical systems (once the physical mechanisms involved are discussed) and it is particularly promising for the simultaneous description of both reversible and irreversible processes in hysteretic media.

\section{ACKNOWLEDGMENTS}

We thank P. P. Delsanto for useful comments and suggestions and acknowledge support from EC (Grant No. FP6502927) and ESF (Grant No. NATEMIS).
*Electronic address: marco.scalerandi@infm.polito.it

${ }^{1}$ J. Tencate, E. Smith, and R. A. Guyer, Phys. Rev. Lett. 85, 1024 (2000).

${ }^{2}$ K. van den Abeele and J. De Vissche, Cem. Concr. Res. 30, 1453 (2000); A. Carpinteri, G. Ferro, and G. Ventura, Eng. Fract. Mech. 70, 995 (2003).

${ }^{3}$ N. Pugno and A. Carpinteri, Trans. ASME, J. Appl. Mech. 23, 832 (2003).

${ }^{4} \mathrm{~K}$. van den Abeele and K. van de Velde, in Review of Progress in $Q N D E$, edited by D. O. Thompson and D. E. Chimenti, AIP Conf. Proc. No. 509 (AIP, Melville, NY, 2000), p. 1359.

${ }^{5}$ R. El-Guerjouma, Adv. Eng. Mater. 3, 601 (2001).

${ }^{6}$ R. A. Guyer and P. A. Johnson, Phys. Today 52, 30 (1999).

${ }^{7}$ H. M. Jaeger, S. R. Nagel, and R. P. Behringer, Phys. Today 49 (4), 32 (1996).

${ }^{8}$ M. Mizuno et al., J. Eur. Ceram. Soc. 18, 1869 (1998); S. Zhu et al., J. Am. Ceram. Soc. 81, 2269 (1998).

${ }^{9}$ C. Caceres, T. Sumitomo, and M. Veidt, Acta Mater. 51, 6211 (2003).

${ }^{10}$ C. Basaran, H. Tang, and S. Nie, Mech. Mater. 36, 1111 (2004).

${ }^{11}$ A. C. Liu, Int. J. Solids Struct. 40, 2121 (2003).

${ }^{12}$ G. Muravskii, Int. J. Solids Struct. 42, 2625 (2005).
${ }^{13}$ I. Etsion, Y. Kligerman, and Y. Kadin, Int. J. Solids Struct. 42, 3716 (2005).

${ }^{14}$ I. D. Mayergoyz, J. Appl. Phys. 57, 3803 (1985).

${ }^{15}$ J. D. Holcombe, J. Geophys. Res. 86, 6235 (1981); K. R. McCall and R. A. Guyer, Nonlinear Processes Geophys. 3, 89 (1996).

${ }^{16}$ M. Scalerandi et al., J. Acoust. Soc. Am. 113, 3049 (2003).

${ }^{17}$ P. P. Delsanto and M. Scalerandi, Phys. Rev. B 68, 064107 (2003).

${ }^{18}$ M. Nobili and M. Scalerandi, Phys. Rev. B 69, 104105 (2004).

${ }^{19}$ B. Capogrosso-Sansone and R. A. Guyer, Phys. Rev. B 66, 224101 (2002).

${ }^{20} \mathrm{~K}$. van den Abeele et al., Ultrasonics 42, 1017 (2004).

${ }^{21}$ V. Gusev and V. Aleshin, J. Acoust. Soc. Am. 112, 2666 (2002);

V. Gusev and V. Yu. Zaitsev, Phys. Lett. A 314, 117 (2003).

${ }^{22}$ J. Kim et al., J. Mech. Phys. Solids 52, 1911 (2004).

${ }^{23}$ C. Pecorari, J. Acoust. Soc. Am. 116, 1939 (2004).

${ }^{24}$ M. W. Barsoum et al., Phys. Rev. Lett. 94, 085501 (2005).

${ }^{25}$ M. Scalerandi, P. P. Delsanto, and P. A. Johnson, J. Phys. D 36, 288 (2003)

${ }^{26}$ M. Bentahar et al., Phys. Rev. B 73, 014116 (2006).

${ }^{27}$ M. Mizuno et al., J. Acoust. Soc. Am. 79, 3065 (1996).

${ }^{28}$ A. G. Evans et al., Acta Metall. Mater. 43, 859 (1995). 